Apala Majumdar

Alain Goriely 



\title{
Static and dynamic stability results for a class of three-dimensional configurations of Kirchhoff elastic rods
}

\author{
Apala Majumdar \& Alain Goriely ${ }^{1}$ \\ OCCAM, Mathematical Institute, University of Oxford, Oxford, OX1 3LB, UK.
}

\begin{abstract}
We analyze the dynamical stability of a naturally straight, inextensible and unshearable elastic rod, under tension and controlled end rotation, within the Kirchhoff model in three dimensions. The cases of clamped boundary conditions and isoperimetric constraints are treated separately. We obtain explicit criteria for the static stability of arbitrary extrema of a general quadratic strain energy. We exploit the equivalence between the total energy and a suitably defined norm to prove that local minimizers of the strain energy, under explicit hypotheses, are stable in the dynamic sense due to Liapounov. We also extend our analysis to damped systems to show that static equilibria are dynamically stable in the Liapounov sense, in the presence of a suitably defined local drag force.
\end{abstract}

Keywords: Elastic Rods; Static Stability; Dynamic Stability; Euler Buckling; Energy Minimizers; Local Drag Models.

\section{Introduction}

Filamentary structures are found in many different systems and diverse applications e.g. biological systems such as DNA, proteins, bacterial fibers; engineering applications such as sub-oceanic cables; theory of liquid crystals and polymers [11, 15, 22]. The stability of filamentary structures is of intrinsic theoretical interest and has important practical implications for the application areas listed above. There are multiple approaches to stability analyses e.g. static bifurcation analysis, dynamic approaches, conjugate point methods, distinguished diagram methods etc. and the interested reader is referred to $[10,16,19,17,21,24]$ for further references.

We focus on the model problem of an intrinsically straight, twisted elastic rod, subject to clamped boundary conditions and a terminal end-load. We only consider a special class of Kirchhoff rods in this paper i.e. an inextensible, unshearable rod with circular cross-section [15]. In our previous work [18], we study the static stability of the undeformed straight state in terms of the second variation of a general quadratic strain energy, in three dimensions. We obtain explicit criteria for the static stability and the onset of instability, in terms of the twist and the tension and our aim, in this paper, is to quantify the dynamic implications

\footnotetext{
${ }^{1}$ Corresponding author: goriely@maths.ox.ac.uk
} 
of static stability criteria for Kirchhoff rods. The intricate relationship between static and dynamic stability is, in general, poorly understood (see [2] for examples of counter-intuitive behaviour) and it is, therefore, of interest to study this relationship for these rods, where both computations can be carried out explicitly.

Our work builds on results reported by Caflisch and Maddocks in [3]. We work in a fully three-dimensional setting i.e. account for both in-plane and out-of-plane configurations and consider two different boundary-value problems: (i) clamped boundary conditions and (ii) clamped boundary conditions with isoperimetric constraints. Our first result is a novel characterization of static equilibria, given by local minimizers of the quadratic strain energy. This characterization follows from an explicit analysis of the integral representation of the second variation of the quadratic strain energy [6]. In particular, our approach is direct without the need to appeal to associated eigenvalue problems. We obtain explicit stability criteria in the Euler angle representation, which in turn yield explicit bounds for the curvature and torsion of the static physically observable equilibria.

The dynamic evolution of the rod is governed by the Kirchhoff equations in three dimensions $[15,16]$ : a six-dimensional system of coupled nonlinear PDEs of second order in space and time, based on linear constitutive relations between moments and Darboux curvature. We do not address questions related to the existence and regularity of solutions of the Kirchhoff equations subject to different boundary conditions as we are mainly interested in the classification of static equilibria and the interplay between static and dynamic stability. The total energy, which is the sum of the kinetic and potential energy, is a conserved quantity $[15,5]$ and following the methods in [3], we establish the equivalence between the energy and a suitably defined dynamic norm in three dimensions. This equivalence allows us to prove the strong statement that local minimizers of the quadratic strain energy (under explicit hypothesis) are stable in the dynamic sense due to Liapounov. More precisely, we use the direct method due to Liapounov to prove the equivalence between static and dynamic stability (under explicit hypothesis) in a three-dimensional framework. This is a significant generalization of the powerful two-dimensional study carried out in [3]. We further extend our results to damped systems with an isotropic drag force proportional to the tangent-velocity; the resulting equations of motion are different to the traditional Kirchhoff equations in [15] and have additional damping terms. However, the total energy is a decreasing function of time and hence, can still be used as a Liapounov function. As a consequence, local minimizers of the strain energy retain dynamic stability in the presence of a suitably defined local drag force. A noteworthy generalization includes the study of arbitrary functionals, that are quadratic and strictly convex with respect to the derivatives of the model variables, wherein we derive explicit criteria that guarantee the equivalence between static and Liapounov stability.

We primarily focus on clamped boundary conditions in this paper and address the question of isoperimetric constraints at the end. In two dimensions, it is known that the classical buckling force for the constrained problem with isoperimetric constraints differs from the classical Euler buckling formula by a factor of 4 [14]. Previous work has demonstrated the onset of instability for terminal loads greater than an explicit critical value. We use Wirtinger's inequality to prove stability in the complementary regime, in two dimensions. In three dimensions, we obtain explicit bounds for the critical tension $F_{c}$ in the presence of isoperimetric 
constraints, such that the unbuckled undistorted state is stable for all loads, $|F|<\left|F_{c}\right|$. Our approach is novel and it is possible that such integral inequalities can be exploited to study post-buckling behaviour too.

The paper is organized as follows. In Section 2, we review the general theory of Kirchhoff rods and elaborate on the concepts of static and dynamic stability. In Section 3, we use Hamilton's principle to derive the equations of motion in terms of the Euler angle representation. In Section 4, we derive explicit static stability criteria and examine the relationship between static and dynamic stability for a clamped Kirchhoff rod and in Section 5, we generalize these results to damped systems. Finally, in Section 6, we consider the case of isoperimetric constraints and in Section 7, we present our conclusions and directions for future work.

\section{Preliminaries}

In this section, we recall the model problem in [18] and the concomitant concepts of static and dynamic Liapounov stability from [3, 17].

The Kirchhoff rod is initially aligned along the $\hat{\mathbf{x}}$-axis of a Cartesian basis, $\{\hat{\mathbf{x}}, \hat{\mathbf{y}}, \hat{\mathbf{z}}\}$, and is subject to an external force, $F$, in the $x$-direction along with a controlled end-rotation at a terminal end [18]. The end-rotation is characterized by a non-zero twist parameter, $M$, throughout the paper. We work in the thin filament approximation and hence, all physically relevant quantities are attached to the central rod-axis. The rod is, thus, parameterized by its arc length, $s$, and since the rod is inextensible, we take $s \in[0,1]$. In a dynamic framework, all dependent variables are functions of $s$ and the time variable, $t \in[0, \infty)$. Following the conventions in [3] and [17], we describe the configuration of the rod by its axis, $\mathbf{r}(s, t)$, and a triad of orthonormal directors $\left\{\mathbf{d}_{1}, \mathbf{d}_{2}, \mathbf{d}_{3}\right\}$ with $\mathbf{d}_{3}$ being the tangent vector, $\mathbf{d}_{3}=\frac{\partial \mathbf{r}}{\partial s}$. The orientation of the basis $\left\{\mathbf{d}_{i}(s, t)\right\}$ changes smoothly relative to a fixed basis $\left\{\mathbf{e}_{i}\right\}$ and this change is described by

$$
\mathbf{d}_{i}^{\prime}=\kappa \times \mathbf{d}_{i} \quad i=1,2,3
$$

where $\mathbf{d}_{i}^{\prime}=\frac{\partial \mathbf{d}_{i}}{d s}$ and

$$
\kappa=\left(\chi_{1}(s, t), \chi_{2}(s, t), \tau(s, t)\right)
$$

is the strain vector. The first two components, $\chi_{1}$ and $\chi_{2}$, contain information about the rod curvature and $\tau$ is a measure of the physical twist.

We follow the Euler angle formulation [5, 17] and introduce a set of Euler angles, $\Theta(s, t)=$ $\{\theta(s, t), \phi(s, t), \psi(s, t)\}$, to describe the orientation of the directors with respect to a fixed basis $\left\{\mathbf{e}_{i}\right\}$. We work with sufficiently smooth rod configurations so that, $\{\theta(s, t), \phi(s, t), \psi(s, t)\}$, are at least twice continuously differentiable i.e. belong to $C^{2}([0,1] \times[0, \infty) ; \mathbb{R})$. Explicit expressions for the directors in the Euler angle representation can be found in $[1,12]$. The strain components are given by [17]

$$
\begin{aligned}
& \chi_{1}=-\phi^{\prime} \sin \theta \cos \psi+\theta^{\prime} \sin \psi \\
& \chi_{2}=\phi^{\prime} \sin \theta \sin \psi+\theta^{\prime} \cos \psi \\
& \tau=\phi^{\prime} \cos \theta+\psi^{\prime} .
\end{aligned}
$$


In what follows, we consider two separate boundary-value problems : (i) BVP I with clamped boundary conditions and (ii) BVP II with clamped boundary conditions and isoperimetric constraints. BVP I is a Dirichlet boundary-value problem for the Euler angles as stated below:

$$
\begin{aligned}
\theta(0) & =\theta(1)=\frac{\pi}{2}, \\
\phi(0) & =\phi(1)=0, \\
\psi(0) & =0 \quad \& \quad \psi(1)=2 \pi M .
\end{aligned}
$$

BVP II is defined by the Dirichlet conditions above and two additional isoperimetric constraints

$$
\begin{array}{ll}
\text { BVP II } & \int_{0}^{1} \sin \theta(s, t) \sin \phi(s, t) d s=0, \\
& \int_{0}^{1} \cos \theta(s, t) d s=0 .
\end{array}
$$

The isoperimetric constraints ensure that the end-point, $s=1$, lies on the $x$-axis and we do not consider the case of self-intersecting rods. The unbuckled state is defined by the triplet

$$
\Theta_{0}=\left(\theta_{0}, \phi_{0}, \psi_{0}\right)=\left(\frac{\pi}{2}, 0,2 \pi M s\right)
$$

that is a straight rod twisted with $M$-turns, aligned along the $x$-axis. It is trivial to note that $\Theta_{0}$ respects both the Dirichlet conditions and the isoperimetric constraints above in (3)-(4).

The Kirchhoff rod, under consideration, is assumed to be hyperelastic, to possess kinetic symmetry and obey linear constitutive stress-strain relations. The potential energy of the rod is then given by $[17,18]$ :

$$
V[\theta, \phi, \psi]:=\int_{0}^{1} \frac{A}{2}\left(\phi_{s}^{2} \sin ^{2} \theta+\theta_{s}^{2}\right)+\frac{C}{2}\left(\phi_{s} \cos \theta+\psi_{s}\right)^{2}+F \sin \theta \cos \phi d s
$$

where $A, C>0$ are material-dependent elastic constants and $F>0$ is the external force. The first two terms in the integrand represent the stored energy density of the rod and the last term is the work done by the external force at the terminal end.

We want to characterize isolated, relative minima of the potential energy in a fully threedimensional framework, accounting for both planar and non-planar equilibria. Here, planar implies that $\theta(s)=\frac{\pi}{2}$ for $s \in[0,1]$ whereas non-planar can account for a non-uniform out-ofplane angle such that, $\theta(s) \neq \frac{\pi}{2}$, for $s$ in some interior interval. Let $\Theta^{*}=\left(\theta^{*}(s), \phi^{*}(s), \psi^{*}(s)\right)$ denote an arbitrary extremal of the potential energy in (6). We further assume that $\theta^{*}(s) \in$ $(0, \pi)$ for all $s \in[0,1]$ i.e. $\theta^{*}$ never encounters the polar singularities at $\theta=0$ and $\theta=\pi$. This is crucial for the stability analysis since the strain energy density loses strict convexity with respect to the gradient arguments at polar singularities and standard results in the calculus of variations do not apply; see [17] and [18] for more details. 
Definition 1. [3] An extremal $\Theta^{*}$ is said to be an isolated strong relative minimum of the potential $V$ in the class of admissible functions if there exists $\epsilon>0$ such that

$$
V\left[\theta^{*}(s)+\alpha(s), \phi^{*}(s)+\beta(s), \psi^{*}(s)+\gamma(s)\right]-V\left[\theta^{*}(s), \phi^{*}(s), \psi^{*}(s)\right]>0
$$

for admissible functions $\alpha, \beta, \gamma:[0,1] \rightarrow \mathbb{R}$ satisfying

$$
0<\sup _{s \in[0,1]}|\alpha(s)|,|\beta(s)|,|\gamma(s)|<\epsilon .
$$

We refer to isolated strong minima of the potential energy as static equilibria in the rest of the text.

From standard results in the calculus of variations [9], we recall that for one-dimensional variational problems as in (6), the positivity of the second variation of the potential energy at an arbitrary extremal, $\Theta^{*}$, is sufficient to guarantee its static stability in the sense defined above $[3,18]$. More precisely, one needs to consider variations of the form

$$
\begin{aligned}
& \theta_{\epsilon}(s)=\theta^{*}(s)+\epsilon \alpha(s) \\
& \phi_{\epsilon}(s)=\phi^{*}(s)+\epsilon \beta(s) \\
& \psi_{\epsilon}(s)=\psi^{*}(s)+\epsilon \gamma(s),
\end{aligned}
$$

where $0<\epsilon \ll 1$ and $\alpha, \beta, \gamma$ are sufficiently smooth functions that vanish at the end-points. For BVP II, the functions $\alpha, \beta, \gamma$ are additionally also constrained to satisfy the linearization of the isoperimetric constraints (4) about the extremal, $\Theta^{*}$, as shown below:

$$
\begin{aligned}
& \int_{0}^{1} \alpha(s) \cos \theta^{*}(s) \sin \phi^{*}(s)+\beta(s) \sin \theta^{*}(s) \cos \phi^{*}(s) d s=0, \\
& \int_{0}^{1} \alpha(s) \sin \theta^{*}(s) d s=0 .
\end{aligned}
$$

One can then explicitly compute the second variation as an integral expression involving $\left\{\theta^{*}, \phi^{*}, \psi^{*}\right\}$ and $\{\alpha, \beta, \gamma\}$ and deduce global conditions for its positivity. We will refer to the positivity of the second variation of the potential energy as the static stability criterion in the subsequent sections.

Whilst studying static stability, we frequently use two versions of Wirtinger's integral inequality as given below [6]:

Proposition 1. (Poincare-Wirtinger inequality) For every continuously differentiable function $u:[0,1] \rightarrow \mathbb{R}$ with $u(0)=u(1)=0$, we have

$$
\int_{0}^{1}\left(\frac{d u}{d s}\right)^{2} d s \geq \pi^{2} \int_{0}^{1} u^{2}(s) d s .
$$

Proposition 2. (Wirtinger inequality) Let

$$
X=\left\{u \in C^{1}([0,1] ; \mathbb{R}) ; u(0)=u(1)=0 ; \quad \int_{0}^{1} u(s) d s=0\right\}
$$


Then

$$
\int_{0}^{1}\left(\frac{d u}{d s}\right)^{2} d s \geq 4 \pi^{2} \int_{0}^{1} u^{2}(s) d s \quad \forall u \in X .
$$

These inequalities are useful for the analysis of the second variation integral and are cited throughout the text.

In [18], we study the second variation of the potential energy about the unbuckled state, $\Theta_{0}$, defined in (5), and obtain results on its stability and instability. In this paper, these results are generalized to an arbitrary extremal point, $\Theta^{*}$, to yield explicit stability criteria in terms of $\theta^{*}, \phi^{*}, \psi^{*}$. Of great interest is the relationship between this static stability criterion and dynamic stability in the Liapounov sense, in a three-dimensional framework. We recall the definition of Liapounov stability from [3].

Definition 2. Let $X$ denote a set of solutions. A solution $\Theta_{1}(s, t) \in X$ is said to be Liapounov stable in $X$, if, for any $\delta>0$, there exists $\epsilon>0$ such that if $\Theta_{2}(s, t) \in X$ satisfies

$$
\left\|\Theta_{1}(s, 0)-\Theta_{2}(s, 0)\right\|<\epsilon
$$

then $\Theta_{2}$ also satisfies

$$
\left\|\Theta_{1}(s, t)-\Theta_{2}(s, t)\right\|<\delta, \quad t \geq 0
$$

where $\|-\|$ is a suitably defined norm.

We take $X$ to be the set of solutions of the equations of motion, derived in Section 3 , subject to BVP I or BVP II in (3) and (4). A suitable norm needs to be defined as stated in (13)-(14); by analogy with the norm in [3], the norm used in this paper is defined to be

$$
\begin{aligned}
& \|\Theta(., t)\|^{2}=\|(\theta(., t), \phi(., t), \psi(., t))\|^{2}= \\
& =\int_{0}^{1} \theta^{2}(s, t)+\phi^{2}(s, t)+\psi^{2}(s, t)+\theta_{s}^{2}(s, t)+\phi_{s}^{2}(s, t)+\psi_{s}^{2}(s, t)+\theta_{t}^{2}(s, t)+\phi_{t}^{2}(s, t)+\psi_{t}^{2}(s, t) d s .
\end{aligned}
$$

Then a solution, $\Theta_{1}=\left(\theta_{1}, \phi_{1}, \psi_{1}\right) \in X$, is Liapounov stable in $X$, if any solution $\Theta_{2}=$ $\left(\theta_{2}, \phi_{2}, \psi_{2}\right) \in X$, which initiates within an $\epsilon$-neighbourhood of $\Theta_{1}$ (see (13)), remains within a small $\delta(\epsilon)$-neighbourhood of $\Theta_{1}$ for all times (see (14)) and $\delta(\epsilon) \rightarrow 0^{+}$as $\epsilon \rightarrow 0^{+}$.

\section{Rod dynamics in Euler angles}

The rod energy is the sum of the potential energy (defined in (6)) and the kinetic energy,

$$
\begin{aligned}
& T[\theta, \phi, \psi]:=\int_{0}^{1} \frac{K_{1}}{2}\left(\phi_{t}^{2} \sin ^{2} \theta+\theta_{t}^{2}\right)+K_{1}\left(\phi_{t} \cos \theta+\psi_{t}\right)^{2} d s+ \\
& +\int_{0}^{1} \frac{\rho}{2}\left(x_{t}^{2}+y_{t}^{2}+z_{t}^{2}\right) d s
\end{aligned}
$$


where

$$
\begin{aligned}
& x(s, t)=\int_{0}^{s} \sin \theta(u, t) \cos \phi(u, t) d u+x(0, t) \\
& y(s, t)=\int_{0}^{s} \sin \theta(u, t) \sin \phi(u, t) d u+y(0, t) \\
& z(s, t)=\int_{0}^{s} \cos \theta(u, t) d u+z(0, t),
\end{aligned}
$$

$K_{1}>0$ is a spin constant and $\rho>0$ is the rod mass-density (mass per unit length) [3, 5]. For simplicity and without loss of generality, we take $x(0, t)=y(0, t)=z(0, t)=0$. Following the methodology in [3] where the authors compute the equations of motion for planar equilibria in a two-dimensional framework, we compute the equations of motion from Hamilton's principle of stationary action i.e. the integral

$$
\int_{t_{0}}^{t_{1}} T[\theta, \phi, \psi]-V[\theta, \phi, \psi] d t
$$

is stationary for all $t_{0}$ and $t_{1}$. For BVP $\mathbf{I},(18)$ is complemented by the following constitutive relations for the resultant force vector, $\mathbf{F}=\left(F^{x}, F^{y}, F^{z}\right)$, where

$$
\begin{aligned}
& F_{s}^{x}=-\rho\left(\int_{0}^{s} \sin \theta(u, t) \cos \phi(u, t) d u\right)_{t t}, \quad F^{x}(1, t)=F>0 ; \\
& F_{s}^{y}=\rho\left(\int_{0}^{s} \sin \theta(u, t) \sin \phi(u, t) d u\right)_{t t}, \quad F^{y}(1, t)=0 ; \\
& F_{s}^{z}=\rho\left(\int_{0}^{s} \cos \theta(u, t) d u\right)_{t t}, \quad F^{z}(1, t)=0 .
\end{aligned}
$$

For BVP II, the Lagrangian in (18) is augmented by two additional Lagrange multipliers accounting for the isoperimetric constraints in (4) as shown below :

$$
\int_{t_{0}}^{t_{1}} T[\theta, \phi, \psi]-V[\theta, \phi, \psi]+\lambda(t) y(1, t)+\nu(t) z(1, t) d t .
$$

Here $\lambda(t)$ and $\nu(t)$ are time-dependent Lagrange multipliers accounting for the constraints in (4) and can be interpreted as being the unknown reaction of the boundary support at $s=1$ that maintains the constraints (4). Following the conventions in [3], the resultant force vector, $\mathbf{F}=\left(F^{x}, F^{y}, F^{z}\right)$, is then given by

$$
\begin{aligned}
& F_{s}^{x}=-\rho\left(\int_{0}^{s} \sin \theta(u, t) \cos \phi(u, t) d u\right)_{t t}, \quad F^{x}(1, t)=F \\
& F_{s}^{y}=\rho\left(\int_{0}^{s} \sin \theta(u, t) \sin \phi(u, t) d u\right)_{t t}, \quad F^{y}(1, t)=\lambda(t) ; \\
& F_{s}^{z}=\rho\left(\int_{0}^{s} \cos \theta(u, t) d u\right)_{t t}, \quad F^{z}(1, t)=\nu(t) .
\end{aligned}
$$


The variational derivation of the corresponding equations of motion is technically difficult but standard. Detailed derivations of the equations of motion can also be found in [5, 15]. We skip the technical details and the equations of motion are given below.

$$
\begin{aligned}
& C\left(\phi_{s} \cos \theta+\psi_{s}\right)_{s}=2 K_{1}\left(\phi_{t} \cos \theta+\psi_{t}\right)_{t} \\
& K_{1} \theta_{t t}+K_{1} \phi_{t}^{2} \sin \theta \cos \theta+2 K_{1} \phi_{t} \psi_{t} \sin \theta= \\
& =A \theta_{s s}-A \phi_{s}^{2} \sin \theta \cos \theta+C \phi_{s} \sin \theta\left(\phi_{s} \cos \theta+\psi_{s}\right)-F^{x} \cos \theta \cos \phi+F^{y} \cos \theta \sin \phi-F^{z} \sin \theta \\
& K_{1} \phi_{t t} \sin \theta-2 K_{1} \theta_{t} \psi_{t}= \\
& =A \phi_{s s} \sin \theta+2 A \phi_{s} \theta_{s} \cos \theta-C \theta_{s}\left(\phi_{s} \cos \theta+\psi_{s}\right)+F^{x} \sin \phi+F^{y} \cos \phi
\end{aligned}
$$

The dynamical system (22) has been derived from Hamilton's principle and hence, the total energy, $E[\theta, \phi, \psi]=T[\theta, \phi, \psi]+V[\theta, \phi, \psi]$, is a constant of motion. In what follows, we use the total energy as a Liapounov function to investigate the relationship between static and dynamic stability criteria.

\section{Stability estimates for BVP I}

Consider the BVP I in (3) with clamped boundary conditions. Our first result concerns the derivation of explicit stability criteria for an arbitrary solution of this boundary-value problem.

\subsection{Static stability}

Proposition 3. Let $\Theta^{*}=\left(\theta^{*}(s), \phi^{*}(s), \psi^{*}(s)\right)$ be an arbitrary extremal of the potential energy (6), subject to the clamped boundary conditions in (3). We assume that $\theta^{*}(s) \in(0, \pi)$ for $s \in[0,1]$ i.e. $\theta^{*}$ remains bounded away from the polar singularities. Then

$$
\phi_{s}^{*} \cos \theta^{*}(s)+\psi_{s}^{*}=K\left(\theta^{*}, \phi^{*}, \psi^{*}\right),
$$

where $K\left(\theta^{*}, \phi^{*}, \psi^{*}\right)$ is a constant that only depends on $\Theta^{*}$. Define the quantities

$$
\begin{aligned}
& \Gamma_{1}=\min _{s \in[0,1]}\left(A\left(\phi_{s}^{*}\right)^{2} \cos 2 \theta^{*}-A\left|\phi_{s}^{*} \sin 2 \theta^{*}\right|-C\left|\phi_{s}^{*} \sin \theta^{*}\right|-C K \phi_{s}^{*} \cos \theta^{*}-C K \sin \theta^{*}\right) \\
& \Gamma_{2}=\min _{s \in[0,1]}\left(A \sin ^{2} \theta^{*}-A\left|\phi_{s}^{*} \sin 2 \theta^{*}\right|-C\left|\cos \theta^{*}\right|-C K \sin \theta^{*}\right) \\
& \Gamma_{3}=\min _{s \in[0,1]}\left(1-\left|\cos \theta^{*}\right|-\left|\phi_{s}^{*} \sin \theta^{*}\right|\right) .
\end{aligned}
$$

Let

$$
\begin{aligned}
& \Gamma_{2}, \Gamma_{3}>0 \\
& A \pi^{2}+\Gamma_{1}>0 .
\end{aligned}
$$

Then $\Theta^{*}$ is stable in the static sense for sufficiently small forces

$$
|F|<\min \left\{\pi^{2} \Gamma_{2}, A \pi^{2}+\Gamma_{1}\right\} .
$$


Comment: Equation (23) is an immediate consequence of the equations of motion (22); for a time-independent solution, we necessarily have $\frac{d}{d s}\left(\phi_{s} \cos \theta+\psi_{s}\right)=0$, which is equivalent to (23).

Proof: We compute the second variation of the potential energy about the extremal, $\Theta^{*}$, by considering arbitrary three-dimensional perturbations of the form (9), where $\epsilon>0$ is a small parameter and $\{\alpha, \beta, \gamma\}$ are continuously differentiable functions that vanish at the end-points, $s=0$ and $s=1$. An explicit computation shows that the second variation of the potential energy about $\Theta^{*}$ is given by

$$
\begin{aligned}
& \delta^{2} V\left(\Theta^{*}\right)=\left.\frac{d^{2}}{d \epsilon^{2}} V\left[\theta_{\epsilon}, \phi_{\epsilon}, \psi_{\epsilon}\right]\right|_{\epsilon=0}= \\
& =\int_{0}^{1} A\left[\beta_{s}^{2} \sin ^{2} \theta^{*}+2 \alpha \beta_{s} \phi_{s}^{*} \sin 2 \theta^{*}+\alpha^{2}\left(\phi_{s}^{*}\right)^{2} \cos 2 \theta^{*}\right] d s+\int_{0}^{1} A \alpha_{s}^{2} d s+ \\
& +\int_{0}^{1} C \gamma_{s}^{2}+C\left(\beta_{s} \cos \theta^{*}-\alpha \phi_{s}^{*} \sin \theta^{*}\right)^{2} d s+\int_{0}^{1} 2 C \gamma_{s} \beta_{s} \cos \theta^{*}-2 C \alpha \gamma_{s} \phi_{s}^{*} \sin \theta^{*} d s- \\
& -2 C K \int_{0}^{1} \alpha \beta_{s} \sin \theta^{*}+\frac{1}{2} \alpha^{2} \phi_{s}^{*} \cos \theta^{*} d s- \\
& -F \int_{0}^{1}\left(\alpha^{2}+\beta^{2}\right) \sin \theta^{*} \cos \phi^{*}+2 \alpha \beta \cos \theta^{*} \sin \phi^{*} d s .
\end{aligned}
$$

We repeatedly use Young's inequality, $a b \leq \frac{1}{2}\left(a^{2}+b^{2}\right)$, to compute a lower bound for $\delta^{2} V\left(\Theta^{*}\right)$ as shown below:

$$
\begin{aligned}
& \delta^{2} V\left(\Theta^{*}\right) \geq \\
& \geq \int_{0}^{1} A \beta_{s}^{2} \sin ^{2} \theta^{*}-A\left|\phi_{s}^{*} \sin 2 \theta^{*}\right|\left(\alpha^{2}+\beta_{s}^{2}\right)+A \alpha^{2}\left(\phi_{s}^{*}\right)^{2} \cos 2 \theta^{*}+A \alpha_{s}^{2} d s+ \\
& +\int_{0}^{1} C \gamma_{s}^{2}-C\left|\cos \theta^{*}\right|\left(\gamma_{s}^{2}+\beta_{s}^{2}\right)-C\left|\phi_{s}^{*} \sin \theta^{*}\right|\left(\alpha^{2}+\gamma_{s}^{2}\right) d s- \\
& -C K \int_{0}^{1}\left[\alpha^{2} \phi_{s}^{*} \cos \theta^{*}+\sin \theta^{*}\left(\alpha^{2}+\beta_{s}^{2}\right)\right] d s-|F| \int_{0}^{1} \alpha^{2}+\beta^{2} d s .
\end{aligned}
$$

Comment: An interesting application of Young's inequality shows that $\left(\alpha^{2}+\beta^{2}\right) \sin \theta^{*} \cos \phi^{*}+2 \alpha \beta \cos \theta^{*} \sin \phi^{*} \leq \frac{1}{2}\left[\left(\alpha^{2}+\beta^{2}\right)\left(\sin ^{2} \theta^{*}+\cos ^{2} \phi^{*}\right)+2|\alpha \beta|\left(\cos ^{2} \theta^{*}+\sin ^{2} \phi^{*}\right)\right]$.

It then suffices to note that

$$
\left(\alpha^{2}+\beta^{2}\right) \sin ^{2} \theta^{*}+2|\alpha \beta| \cos ^{2} \theta^{*} \leq \alpha^{2}+\beta^{2}
$$

to deduce that

$$
\left(\alpha^{2}+\beta^{2}\right) \sin \theta^{*} \cos \phi^{*}+2 \alpha \beta \cos \theta^{*} \sin \phi^{*} \leq\left(\alpha^{2}+\beta^{2}\right)
$$


Recalling the definitions of $\Gamma_{1}, \Gamma_{2}, \Gamma_{3}$ from (24) and grouping together the coefficients of $\{\alpha, \beta, \gamma\}$ and their first derivatives in (28), we obtain

$$
\delta^{2} V\left(\Theta^{*}\right) \geq \int_{0}^{1} A \alpha_{s}^{2}+\alpha^{2}\left(\Gamma_{1}-|F|\right) d s+\int_{0}^{1} \Gamma_{2} \beta_{s}^{2}-|F| \beta^{2} d s+\int_{0}^{1} C \Gamma_{3} \gamma_{s}^{2} d s .
$$

Let $|F|<\min \left\{\pi^{2} \Gamma_{2}, A \pi^{2}+\Gamma_{1}\right\}$. We focus on the first term on the right-hand side of (29) and compute conditions which guarantee the positivity of this integral. If $\Gamma_{1} \geq|F|$, then the integral, $\int_{0}^{1} A \alpha_{s}^{2}+\alpha^{2}\left(\Gamma_{1}-|F|\right) d s$, is necessarily positive and there is nothing to prove. We assume that $\Gamma_{1}<|F|$. Then the first integral on the right-hand side of (29) can be decomposed as

$$
\int_{0}^{1} A \alpha_{s}^{2}+\alpha^{2}\left(\Gamma_{1}-|F|\right) d s=\int_{0}^{1}\left(A+\frac{\Gamma_{1}-|F|}{\pi^{2}}\right) \alpha_{s}^{2}+\left(|F|-\Gamma_{1}\right)\left(\frac{\alpha_{s}^{2}}{\pi^{2}}-\alpha^{2}\right) d s .
$$

Since $\Gamma_{1}<|F|$ by assumption, we can use Wirtinger's inequality (11) to note that

$$
\left(|F|-\Gamma_{1}\right) \int_{0}^{1}\left(\frac{\alpha_{s}^{2}}{\pi^{2}}-\alpha^{2}\right) d s \geq 0
$$

and hence

$$
\int_{0}^{1} A \alpha_{s}^{2}+\alpha^{2}\left(\Gamma_{1}-|F|\right) d s \geq \int_{0}^{1}\left(A+\frac{\Gamma_{1}-|F|}{\pi^{2}}\right) \alpha_{s}^{2} d s
$$

where $\left(A+\frac{\Gamma_{1}-|F|}{\pi^{2}}\right)>0$ from the hypothesis.

Using similar arguments as above, we recast the integral on the right-hand side of (29) as

$$
\delta^{2} V\left(\Theta^{*}\right) \geq \min \left\{\min \left\{A, A+\frac{\Gamma_{1}-|F|}{\pi^{2}}\right\}, \Gamma_{2}-\frac{|F|}{\pi^{2}}, C \Gamma_{3}\right\} \int_{0}^{1} \alpha_{s}^{2}+\beta_{s}^{2}+\gamma_{s}^{2} d s .
$$

Hence, if

$$
\min \left\{\min \left\{A, A+\frac{\Gamma_{1}-|F|}{\pi^{2}}\right\}, \Gamma_{2}-\frac{|F|}{\pi^{2}}, C \Gamma_{3}\right\}>0
$$

from the hypotheses (25) and (26), then

$$
\delta^{2} V\left(\Theta^{*}\right)>0
$$

leading to the conclusion of Proposition 3.

Comment: We note that in general, $\Theta^{*}$ depends on $A, C, M$ and the external load $F$. An exception is the unbuckled state, $\Theta_{0}$, which is an equilibrium for all $F>0$. Hence, the criterion (26) specifies a self-consistent relation between these quantities that guarantees static stability. 


\subsection{Interpretation of the static stability criterion}

We give an interpretation of the bounds (25) in terms of the curvature and torsion of the static equilibria. The strain components can be equivalently expressed as

$$
\begin{aligned}
& \chi_{1}=\kappa \sin \Delta \\
& \chi_{2}=\kappa \cos \Delta \\
& \tau=\eta+\Delta_{s}
\end{aligned}
$$

where $\kappa$ is the curvature, $\eta$ is the torsion and $\Delta$ is a register variable [4]. In terms of the Euler angles, we compare (33) with (2) to find

$$
\begin{aligned}
& \theta_{s}=\kappa \cos \psi \\
& \phi_{s} \sin \theta=\kappa \sin \psi \\
& \psi_{s}=\eta-\phi_{s} \cos \theta .
\end{aligned}
$$

We consider the bounds (25) for solutions with constant curvature, $\kappa$, and torsion, $\eta$ (setting $\Delta(s)=0$ ), without paying attention to the boundary conditions. A qualitative analysis shows that the bounds (25) require

$$
\begin{aligned}
& \kappa \leq 1 ; \quad \eta \leq \frac{A}{C} \\
& \min _{s \in[0,1]} \sin ^{2} \theta^{*}(s) \geq\left\{\frac{C^{2} \eta^{2}}{A^{2}}, \max _{s \in[0,1]}\left\{\frac{C}{A}, 2 \kappa\right\}\left|\cos \theta^{*}\right|\right\} \\
& \frac{C}{A} \leq\left(\frac{\pi^{2}}{\kappa}+2 \kappa\right) .
\end{aligned}
$$

These bounds are not rigorous and are meant to give general insight in terms of geometric quantities. Loosely speaking, they require that the curvature and torsion be small and that the equilibrium remains sufficiently bounded away from the polar singularities i.e. $\sin ^{2} \theta^{*}$ is always bounded from below. Recalling the underlying assumptions of rod-theory [1, 12], the curvature and torsion are always necessarily small compared to geometrical parameters and $\frac{2}{3} \leq \frac{C}{A} \leq 1$ [15]. Hence, these bounds do not impose unrealistic restrictions on the curvature, torsion and material-dependent parameters. Although, we need to perform a deeper analysis to understand the implications of (25) for buckled equilibria, the bounds (25) and (26) provide a set of explicit stability criteria relating the end-rotation, terminal load, material constants and the equilibrium itself. In principle, one could numerically compute equilibria and easily check their stability against these explicit criteria.

We can compute the quantities (24) for the unbuckled state, $\Theta_{0}$, defined in (5). They are $K=2 \pi M, \Gamma_{2}=A-2 \pi M C$ and $\Gamma_{3}=1$ from the definitions in (24). Then $\Theta_{0}$ is stable for forces

$$
|F|<\min \left\{\pi^{2}(A-2 \pi M C), A \pi^{2}-2 \pi M C\right\}
$$

from the stability criteria in (26), which is in perfect agreement with the results in [18]. In [18], we demonstrate that the undeformed straight state is stable for forces

$$
|F|<\min \left\{\pi^{2}(A-2 \pi M C), A \pi^{2}-2 \pi M C\right\}
$$


and is unstable for forces $|F|>A \pi^{2}$. Our next propositions concern the equivalence between static and dynamic Liapounov stability for static equilibria.

\subsection{Dynamic Liapounov Stability}

Proposition 4. The unbuckled equilibrium, $\Theta_{0}$, defined in (5), is Liapounov stable in the set of solutions, $\Theta^{*}(s, t)=\left(\theta^{*}(s, t), \phi^{*}(s, t), \psi^{*}(s, t)\right) \in C^{2}([0,1] \times[0, \infty) ; \mathbb{R})$, of the dynamical system (22) subject to the boundary conditions (3), for forces

$$
|F|<\min \left\{\pi^{2}(A-2 \pi M C), A \pi^{2}-2 \pi M C\right\} .
$$

Here, Liapounov stability is understood in terms of the norm defined in (15).

Proof: Let $\Theta^{*}(s, t)$ be an arbitrary solution of the dynamical system (22). One can readily check that the unbuckled state, $\Theta_{0}$, is a solution of the dynamical system (22) subject to the boundary conditions (3). Define the difference, $\Theta_{1}(s, t)=\Theta^{*}(s, t)-\Theta_{0}(s)=\left(\theta_{1}, \phi_{1}, \psi_{1}\right)$ i.e.

$$
\begin{aligned}
& \theta_{1}(s, t)=\theta^{*}(s, t)-\theta_{0}(s) \\
& \phi_{1}(s, t)=\phi^{*}(s, t)-\phi_{0}(s) \\
& \psi_{1}(s, t)=\psi^{*}(s, t)-\psi_{0}(s) ;
\end{aligned}
$$

the clamped boundary conditions in (3) necessarily imply that the triplet $\Theta_{1}$ vanishes at the end-points. We consider solutions that initiate in a small $\epsilon$-neighbourhood of $\Theta_{0}$ for which

$$
\left\|\Theta_{1}(., 0)\right\|^{2}<\epsilon ;
$$

recall the definition of the norm in (15). To demonstrate Liapounov stability, we need to prove that the norm $\left\|\Theta_{1}(., t)\right\|^{2}$ remains small for all subsequent times.

In what follows, we study the second variation of the potential energy, $V[\theta, \phi, \psi]$, around $\Theta_{0}$, exploit relations between the potential energy and its second variation and finally use the conservation of energy

$$
\Delta E(t)=E\left[\Theta^{*}(., t)\right]-E\left[\Theta_{0}\right]=\Delta E(0)
$$

to establish Liapounov stability for forces $|F|<\min \left\{\pi^{2}(A-2 \pi M C), A \pi^{2}-2 \pi M C\right\}$.

In $[18]$, we compute the second variation of the potential energy, $\delta^{2} V\left[\Theta_{0}\right]$, around $\Theta_{0}$ to be

$$
\delta^{2} V\left[\Theta_{0}\right]=A \int_{0}^{1}\left(\theta_{1 s}^{2}+\phi_{1 s}^{2}\right) d s+\int_{0}^{1} C \psi_{1 s}^{2}-4 \pi M C \theta_{1} \phi_{1 s}-|F|\left(\theta_{1}^{2}+\phi_{1}^{2}\right) d s .
$$

Since $|F|<\min \left\{\pi^{2}(A-2 \pi M C), A \pi^{2}-2 \pi M C\right\}$, we decompose the second variation integral in (40) as follows:

$$
\begin{aligned}
& \delta^{2} V\left[\Theta_{0}\right] \geq \int_{0}^{1}\left[A-\frac{|F|+2 \pi M C}{\pi^{2}}\right] \theta_{1 s}^{2}+\left(\frac{|F|+2 \pi M C}{\pi^{2}}\right)\left(\theta_{1 s}^{2}-\pi^{2} \theta_{1}^{2}\right) d s+ \\
& +\int_{0}^{1}\left[A-2 \pi M C-\frac{|F|}{\pi^{2}}\right] \phi_{1 s}^{2} d s+\frac{|F|}{\pi^{2}}\left(\phi_{1 s}^{2}-\pi^{2} \phi_{1}^{2}\right) d s+ \\
& +\int_{0}^{1} C \psi_{1 s}^{2} d s .
\end{aligned}
$$


From Wirtinger's inequality (11),

$$
\int_{0}^{1}\left(\theta_{1 s}^{2}-\pi^{2} \theta_{1}^{2}\right) d s \geq 0
$$

and from the hypotheses, $\min \left\{A-\frac{|F|+2 \pi M C}{\pi^{2}}, A-2 \pi M C-\frac{|F|}{\pi^{2}}\right\}>0$. Hence, $\delta^{2} V\left[\Theta_{0}\right]$ is bounded from below by

$$
\delta^{2} V\left[\Theta_{0}\right] \geq \min \left\{A-\frac{|F|+2 \pi M C}{\pi^{2}}, A-2 \pi M C-\frac{|F|}{\pi^{2}}, C\right\} \int_{0}^{1} \theta_{1 s}^{2}+\phi_{1 s}^{2}+\psi_{1 s}^{2} d s
$$

We recall (11) and use the inequality

$$
\int_{0}^{1} \theta_{1 s}^{2} d s \geq \frac{1}{2} \int_{0}^{1} \theta_{1 s}^{2}+\theta_{1}^{2} d s
$$

for functions, $\theta_{1} \in C^{1}([0,1] ; \mathbb{R})$, such that $\theta_{1}(0)=\theta_{1}(1)=0$. Therefore,

$$
\begin{aligned}
& \delta^{2} V\left[\Theta_{0}\right] \geq \frac{1}{2} \min \left\{A-\frac{|F|+2 \pi M C}{\pi^{2}}, A-2 \pi M C-\frac{|F|}{\pi^{2}}, C\right\} \times \\
& \times \int_{0}^{1} \theta_{1 s}^{2}+\phi_{1 s}^{2}+\psi_{1 s}^{2}+\theta_{1}^{2}+\phi_{1}^{2}+\psi_{1}^{2} d s .
\end{aligned}
$$

It is straightforward to verify that

$$
\delta^{2} V\left[\Theta_{0}\right] \leq \max \{A, A+2 \pi M C, C\} \int_{0}^{1} \theta_{1 s}^{2}+\phi_{1 s}^{2}+\psi_{1 s}^{2}+\theta_{1}^{2}+\phi_{1}^{2}+\psi_{1}^{2} d s .
$$

From (44) and (45), we deduce the existence of two positive constants $a_{1}$ and $a_{2}$, independent of $\Theta_{1}$, such that

$$
\begin{aligned}
& a_{1} \int_{0}^{1} \theta_{1 s}^{2}+\phi_{1 s}^{2}+\psi_{1 s}^{2}+\theta_{1}^{2}+\phi_{1}^{2}+\psi_{1}^{2} d s \leq \delta^{2} V\left[\Theta_{0}\right] \leq \\
& \leq a_{2} \int_{0}^{1} \theta_{1 s}^{2}+\phi_{1 s}^{2}+\psi_{1 s}^{2}+\theta_{1}^{2}+\phi_{1}^{2}+\psi_{1}^{2} d s .
\end{aligned}
$$

From [18], it is known that $\Theta_{0}$ is a strong potential energy minimum under the hypothesis (36). Following the methods in [3], the potential energy close to a local minimum can be bounded by its second variation as follows:

$$
a_{3} \delta^{2} V\left[\Theta_{0}\right] \leq \Delta V=V\left[\theta^{*}, \phi^{*}, \psi^{*}\right]-V\left[\theta_{0}, \phi_{0}, \psi_{0}\right] \leq a_{4} \delta^{2} V\left[\Theta_{0}\right]
$$

where $a_{3}, a_{4}$ are positive constants that only depend on $A, C,|F|$ and the twist parameter $M>0$.

The next step is to compute lower and upper bounds for the kinetic energy difference

$$
\Delta T=T\left[\theta^{*}, \phi^{*}, \psi^{*}\right]-T\left[\theta_{0}, \phi_{0}, \psi_{0}\right]
$$


where $T$ has been defined in (16). The kinetic energy of $\Theta_{0}$ vanishes since this is a timeindependent solution. Thus,

$$
\begin{aligned}
& T\left[\theta^{*}, \phi^{*}, \psi^{*}\right]=\int_{0}^{1} \frac{K_{1}}{2}\left(\theta_{1 t}^{2}+\phi_{1 t}^{2} \sin ^{2} \theta^{*}(s, t)+2\left(\phi_{1 t} \cos \theta^{*}(s, t)+\psi_{1 t}\right)^{2}\right) d s+ \\
& +\int_{0}^{1} \frac{\rho}{2}\left[\left(\int_{0}^{s}\left(\sin \theta^{*} \cos \phi^{*}\right)_{t} d s\right)^{2}+\left(\int_{0}^{s}\left(\sin \theta^{*} \sin \phi^{*}\right)_{t} d s\right)^{2}+\left(\int_{0}^{s}\left(\cos \theta^{*}\right)_{t} d s\right)^{2}\right] d s
\end{aligned}
$$

and $\theta_{1}, \phi_{1}$ have been defined in (37). Since we are only considering solutions, $\Theta^{*}$, in a neighbourhood of $\Theta_{0}$, the inequality, $\sin ^{2} \theta^{*} \geq \frac{1}{2}+\cos ^{2} \theta^{*}$, holds for all $t \geq 0$ and $s \in[0,1]$. Thus,

$$
\begin{aligned}
& \frac{K_{1}}{2}\left(\theta_{1 t}^{2}+\frac{1}{2} \phi_{1 t}^{2}+\frac{2}{3} \psi_{1 t}^{2}\right) \leq \frac{K_{1}}{2}\left(\theta_{1 t}^{2}+\phi_{1 t}^{2} \sin ^{2} \theta^{*}(s, t)+2\left(\phi_{1 t} \cos \theta^{*}(s, t)+\psi_{1 t}\right)^{2}\right) \leq \\
& \leq \frac{K_{1}}{2}\left(\theta_{1 t}^{2}+5 \phi_{1 t}^{2}+4 \psi_{1 t}^{2}\right) .
\end{aligned}
$$

Similarly, one can check that

$$
\begin{aligned}
& \frac{\rho}{2} \int_{0}^{1}\left[\left(\int_{0}^{s}\left(\sin \theta^{*} \cos \phi^{*}\right)_{t} d s\right)^{2}+\left(\int_{0}^{s}\left(\sin \theta^{*} \sin \phi^{*}\right)_{t} d s\right)^{2}+\left(\int_{0}^{s}\left(\cos \theta^{*}\right)_{t} d s\right)^{2}\right] d s \leq \\
& \leq \frac{\rho}{2} \int_{0}^{1} \theta_{1 t}^{2}+\phi_{1 t}^{2} d s .
\end{aligned}
$$

Combining (50) and (51), we deduce that there are two positive constants, $a_{5}, a_{6}$, depending only on $K_{1}$ and $\rho$, such that

$$
a_{5} \int_{0}^{1} \theta_{1 t}^{2}+\phi_{1 t}^{2}+\psi_{1 t}^{2} d s \leq \Delta T \leq a_{6} \int_{0}^{1} \theta_{1 t}^{2}+\phi_{1 t}^{2}+\psi_{1 t}^{2} d s .
$$

From (46), (47) and (52), the total energy difference satisfies the inequalities

$$
a_{7}\left\|\Theta_{1}(., t)\right\|^{2} \leq \Delta E(t)=\Delta V(t)+\Delta T(t) \leq a_{8}\left\|\Theta_{1}(., t)\right\|^{2},
$$

$a_{7}, a_{8}$ are positive constants independent of $\Theta_{1}$, and the norm $\|-\|^{2}$ has been defined in (15). However, $\Delta E(t)=\Delta E(0)$ (from conservation of energy) and from the inequalities in (53),

$$
\Delta E(0) \leq a_{8}\left\|\Theta_{1}(., 0)\right\|^{2}<a_{8} \epsilon
$$

as a consequence of the imposed initial condition (see (38)). Thus,

$$
\left\|\Theta_{1}(., t)\right\|^{2} \leq \frac{a_{8}}{a_{7}} \epsilon
$$

for all $t \geq 0$. Hence, the norm $\|-\|^{2}$ remains small for all subsequent times and we conclude that $\Theta_{0}$ is Liapounov stable under the hypothesis (36).

Following the characterization of static equilibria in Proposition 3, we next address the question of their dynamic stability. 
Proposition 5. Let $\Theta^{*}=\left(\theta^{*}(s), \phi^{*}(s), \psi^{*}(s)\right)$ be an arbitrary extremal of the potential energy (6), subject to the clamped boundary conditions in (3), such that $\theta^{*} \in(0, \pi)$ for $s \in[0,1]$. Then $\Theta^{*}$ is Liapounov stable in the set of $C^{2}$-solutions of the dynamical system (22), with respect to the norm defined in (15), if

$$
\begin{aligned}
& \Gamma_{2}=\min _{s \in[0,1]}\left(A \sin ^{2} \theta^{*}-A\left|\phi_{s}^{*} \sin 2 \theta^{*}\right|-C\left|\cos \theta^{*}\right|-C K \sin \theta^{*}\right)>0 \\
& \Gamma_{3}=\min _{s \in[0,1]}\left(1-\left|\cos \theta^{*}\right|-\left|\phi_{s}^{*} \sin \theta^{*}\right|\right)>0 \\
& \Gamma_{1}=\min _{s \in[0,1]}\left(A\left(\phi_{s}^{*}\right)^{2} \cos 2 \theta^{*}-A\left|\phi_{s}^{*} \sin 2 \theta^{*}\right|-C\left|\phi_{s}^{*} \sin \theta^{*}\right|-C K \phi_{s}^{*} \cos \theta^{*}-C K \sin \theta^{*}\right)>-A \pi^{2}
\end{aligned}
$$

and for applied forces

$$
|F|<\min \left\{\pi^{2} \Gamma_{2}, A \pi^{2}+\Gamma_{1}\right\} .
$$

Proof: In Proposition 3, we show that under the hypotheses (55) and (56), the second variation of the potential energy about $\Theta^{*}$ is bounded from below by

$$
\delta^{2} V\left(\Theta^{*}\right) \geq \min \left\{\min \left\{A, A+\frac{\Gamma_{1}-|F|}{\pi^{2}}\right\}, \Gamma_{2}-\frac{|F|}{\pi^{2}}, C \Gamma_{3}\right\} \int_{0}^{1} \alpha_{s}^{2}+\beta_{s}^{2}+\gamma_{s}^{2} d s
$$

Equivalently, from (11) (using arguments similar to (43)),

$$
\begin{aligned}
& \delta^{2} V\left(\Theta^{*}\right) \geq \frac{1}{2} \min \left\{\min \left\{A, A+\frac{\Gamma_{1}-|F|}{\pi^{2}}\right\}, \Gamma_{2}-\frac{|F|}{\pi^{2}}, C \Gamma_{3}\right\} \times \\
& \times \int_{0}^{1} \alpha_{s}^{2}+\beta_{s}^{2}+\gamma_{s}^{2}+\alpha^{2}+\beta^{2}+\gamma^{2} d s
\end{aligned}
$$

for arbitrary $C^{1}$-functions, $\bar{\Theta}_{1}=(\alpha, \beta, \gamma)$, that vanish at the terminal points. Similarly, there exists a positive constant, $a_{9}$, independent of $\bar{\Theta}_{1}$, such that (see (45) for analogous arguments in the case of $\left.\Theta_{0}\right)$

$$
\delta^{2} V\left(\Theta^{*}\right) \leq a_{9} \int_{0}^{1} \alpha_{s}^{2}+\beta_{s}^{2}+\gamma_{s}^{2}+\alpha^{2}+\beta^{2}+\gamma^{2} d s
$$

From (58) and (59), there exist two positive constants $a_{9}$ and $a_{10}$ (independent of $\bar{\Theta}_{1}$ ) such that

$$
\begin{aligned}
& a_{10} \int_{0}^{1} \alpha_{s}^{2}+\beta_{s}^{2}+\gamma_{s}^{2}+\alpha^{2}+\beta^{2}+\gamma^{2} d s \leq \delta^{2} V\left(\Theta^{*}\right) \leq \\
& \leq a_{9} \int_{0}^{1} \alpha_{s}^{2}+\beta_{s}^{2}+\gamma_{s}^{2}+\alpha^{2}+\beta^{2}+\gamma^{2} d s .
\end{aligned}
$$

This is analogous to the inequality (46) in Proposition 4, with $\bar{\Theta}_{1}$ being identified with $\Theta_{1}$ in (37). 
The rest of the proof parallels that of Proposition 4. Let

$$
\bar{\Theta}^{*}(s, t)=\Theta^{*}(s)+\bar{\Theta}_{1}(s, t)
$$

denote an arbitrary solution of the dynamical system (22) subject to the boundary conditions (3), confined to a small neighborhood of $\Theta^{*}$, such that

$$
\left\|\bar{\Theta}_{1}(., 0)\right\|^{2}<\epsilon
$$

and $\|-\|^{2}$ has been defined in (15). There are two key inequalities:

$$
\begin{aligned}
& a_{11} \delta^{2} V\left(\Theta^{*}\right) \leq \Delta V=V\left(\bar{\Theta}^{*}(s, t)\right)-V\left(\Theta^{*}(s)\right) \leq a_{12} \delta^{2} V\left(\Theta^{*}\right) \\
& a_{13} \int_{0}^{1} \alpha_{t}^{2}+\beta_{t}^{2}+\gamma_{t}^{2} d s \leq \Delta T=T\left(\bar{\Theta}^{*}(s, t)\right) \leq a_{14} \int_{0}^{1} \alpha_{t}^{2}+\beta_{t}^{2}+\gamma_{t}^{2} d s,
\end{aligned}
$$

the derivation of the second inequality (involving the kinetic energy) is identical to (52) and all constants $a_{11} \ldots a_{14}$ are independent of $\bar{\Theta}_{1}(s, t)$. Proposition 5 follows from combining the inequalities, (60) and (61), above with the conservation of energy.

\subsection{Generalizations}

We conclude this section by generalizing our methods to an energy functional with $n$ dependent variables, $y_{1} \cdots y_{n}$, and all $n$ dependent variables are assumed to be smooth on the real interval, $s \in[0,1]$. We take

$$
E\left[y_{1} \cdots y_{n}\right]:=\int_{0}^{1} V\left[y_{1} \cdots y_{n}, y_{1}^{\prime} \cdots y_{n}^{\prime}\right]+g\left[y_{1} \cdots y_{n}\right] d s
$$

$y_{i}^{\prime}=\frac{d y_{i}}{d s}$, the potential energy $V$ is quadratic in the gradient arguments and is strictly convex with respect to the gradient arguments in the sense that

$$
\sum_{i, j=1}^{n} \eta_{i}^{\prime} \eta_{j}^{\prime} V_{y_{i}^{\prime} y_{j}^{\prime}} \geq \alpha \sum_{i=1}^{n}\left(\frac{d \eta_{i}}{d s}\right)^{2}
$$

where $\left\{\eta_{1} \ldots \eta_{n}\right\} \in C^{\infty}([0,1] ; \mathbb{R})$ and $\alpha>0$ is a positive constant independent of the $\eta_{i}$ 's [7].

Let $\left\{y_{i}^{*}\right\}$ denote an arbitrary extremal of the functional in (62), subject to Dirichlet boundary conditions. Since this is a one-dimensional variational problem, Weierstrass's theory guarantees the static stability of $\left\{y_{i}^{*}\right\}$ under the positivity of the second variation of $E\left[y_{1} \cdots y_{n}\right]$ around $\left\{y_{i}^{*}\right\}$. As in Proposition 3, we consider perturbations of the form

$$
y_{\epsilon i}(s)=y_{i}(s)+\epsilon \eta_{i}(s) \quad i=1 \cdots n,
$$

where $\epsilon>0$ is a small parameter and the functions, $\eta_{i}$, vanish at the end-points. The second variation, $\delta^{2} E\left(\left\{y_{i}^{*}\right\}\right)$, can be explicitly computed as a Taylor expansion as shown below:

$$
\begin{aligned}
& \delta^{2} E\left(\left\{y_{i}^{*}\right\}\right)=\left.\sum_{i, j=1}^{n} \int_{0}^{1} \eta_{i} \eta_{j}\left[\frac{\partial^{2} V}{\partial y_{i} \partial y_{j}}+\frac{\partial^{2} g}{\partial y_{i} \partial y_{j}}\right]\right|_{\left\{y_{i}^{*}\right\}} d s+ \\
& +\left.\sum_{i, j=1}^{n} \int_{0}^{1}\left[\eta_{i}^{\prime} \eta_{j}^{\prime} \frac{\partial^{2} V}{\partial y_{i}^{\prime} \partial y_{j}^{\prime}}+\eta_{i} \eta_{j}^{\prime} \frac{\partial^{2} V}{\partial y_{i} \partial y_{j}^{\prime}}\right]\right|_{\left\{y_{i}^{*}\right\}} d s .
\end{aligned}
$$


A lower bound for (65) can be obtained from the following inequalities:

$$
\begin{aligned}
& \left.\sum_{i, j=1}^{n} \eta_{i}^{\prime} \eta_{j}^{\prime} \frac{\partial^{2} V}{\partial y_{i}^{\prime} \partial y_{j}^{\prime}}\right|_{\left\{y_{i}^{*}\right\}} \geq \alpha \sum_{i=1}^{n}\left(\frac{d \eta_{i}}{d s}\right)^{2} \\
& \left.\sum_{i, j=1}^{n} \eta_{i} \eta_{j}\left[\frac{\partial^{2} V}{\partial y_{i} \partial y_{j}}+\frac{\partial^{2} g}{\partial y_{i} \partial y_{j}}\right]\right|_{\left\{y_{i}^{*}\right\}} \geq \sum_{i=1}^{n} \eta_{i}^{2}\left[V_{y_{i} y_{i}}+g_{y_{i} y_{i}}-\sum_{j \neq i}\left|V_{y_{i} y_{j}}\right|+\left|g_{y_{i} y_{j}}\right|\right] \\
& \left.\left.\sum_{i, j=1}^{n} \eta_{i} \eta_{j}^{\prime} \frac{\partial^{2} V}{\partial y_{i} \partial y_{j}^{\prime}}\right|_{\left\{y_{i}^{*}\right\}} \geq-\sum_{i=1}^{n}\left[\left|V_{y_{i} y_{i}^{\prime}}\right|_{y_{i}^{*}}\left(\eta_{i}^{2}+\left(\eta_{i}^{\prime}\right)^{2}\right)+\frac{1}{2} \sum_{j \neq i}\left|V_{y_{i} y_{j}^{\prime}}\right|_{y_{i}^{*}}\left(\eta_{i}^{2}+\left(\eta_{j}^{\prime}\right)^{2}\right)\right] 66\right)
\end{aligned}
$$

Define the following functions:

$$
\begin{aligned}
& \Gamma_{1 i}\left(\left\{y_{i}^{*}\right\}\right)=\min _{s \in[0,1]}\left[\alpha-\left|V_{y_{i} y_{i}^{\prime}}\right|_{y_{i}^{*}}-\frac{1}{2} \sum_{j \neq i}\left|V_{y_{i}^{\prime} y_{j}}\right|_{y_{i}^{*}}\right] \\
& \Gamma_{2 i}\left(\left\{y_{i}^{*}\right\}\right)=\min _{s \in[0,1]}\left(V_{y_{i} y_{i}}+g_{y_{i} y_{i}}-\left|V_{y_{i} y_{i}^{\prime}}\right|_{y_{i}^{*}}-\sum_{j \neq i}\left[\left|V_{y_{i} y_{j}}\right|+\left|g_{y_{i} y_{j}}\right|\right]_{y_{i}^{*}}+\frac{1}{2}\left|V_{y_{i} y_{j}^{\prime}}\right|_{y_{i}^{*}}\right)
\end{aligned}
$$

Substituting (66) and (67) into (65) shows that

$$
\delta^{2} E\left(\left\{y_{i}^{*}\right\}\right) \geq \sum_{i=1}^{n} \int_{0}^{1} \Gamma_{1 i}\left(\frac{d \eta_{i}}{d s}\right)^{2}+\Gamma_{2 i} \eta_{i}^{2}(s) d s .
$$

One can immediately deduce that $\delta^{2} E>0$ if

$$
\begin{aligned}
& \Gamma_{1 i}>0 \text { for } i=1 \ldots n \text { and } \\
& \pi^{2} \Gamma_{1 i}+\Gamma_{2 i}>0 \text { for } i=1 \ldots n .
\end{aligned}
$$

The inequality $\pi^{2} \Gamma_{1 i}+\Gamma_{2 i}>0$ follows from Wirtinger's inequality

$$
\int_{0}^{1}\left(\frac{d \eta_{i}}{d s}\right)^{2} \geq \pi^{2} \int_{0}^{1} \eta_{i}^{2}(s) d s
$$

since $\eta_{i}(0)=\eta_{i}(1)=0$ by virtue of the clamped boundary conditions.

The constraints (69) are a set of explicit stability criteria for extrema of the energy functional (62). The equivalence between the stability criteria in (69) and dynamic Liapounov stability with respect to a suitably defined norm, follows by our previous methodology.

\section{Damped systems}

In this section, we consider a local drag force, $\mathbf{F}_{D}$, acting on the elastic rod, given by

$$
\mathbf{F}_{D} \propto-\xi(s)\left([\sin \theta \cos \phi]_{t},[\sin \theta \sin \phi]_{t},[\cos \theta]_{t}\right)
$$


where $\xi(s)>0$ is an inhomogeneous, positive damping coefficient. As a consequence,

$$
\mathbf{d}_{3} \cdot \mathbf{F}_{D}=(\sin \theta \cos \phi, \sin \theta \sin \phi, \cos \theta) \cdot \mathbf{F}_{D}=0
$$

since $\mathbf{d}_{3} \cdot \mathbf{d}_{3}=1$ i.e. the drag force is acting normal to the tangent vector of the rod. Our aim is to study dynamic stability of local minimizers of the potential energy in (6), in the presence of such local damping.

The resultant force vector, $\mathbf{F}=\left(F^{x}, F^{y}, F^{z}\right)$, is given by the following constitutive relations that include the additional damping terms as shown below:

$$
\begin{aligned}
& F_{s}^{x}=-\rho\left(\int_{0}^{s} \sin \theta \cos \phi d u\right)_{t t}-[\xi(s) \sin \theta \cos \phi]_{s t} \\
& F_{s}^{y}=\rho\left(\int_{0}^{s} \sin \theta \sin \phi d u\right)_{t t}+[\xi(s) \sin \theta \sin \phi]_{s t} \\
& F_{s}^{z}=\rho\left(\int_{0}^{s} \cos \theta d u\right)_{t t}+[\xi(s) \cos \theta]_{s t},
\end{aligned}
$$

coupled with the boundary conditions

$$
F^{x}(1, t)=F>0 ; \quad F^{y}(1, t)=F^{z}(1, t)=0 .
$$

The clamped boundary conditions in (3) necessarily imply that

$$
[\sin \theta \cos \phi]_{t}=[\sin \theta \sin \phi]_{t}=[\cos \theta]_{t}=0
$$

at the terminal points, $s=0$ and $s=1$.

The corresponding equations of motion for the Euler angles are:

$$
\begin{aligned}
& C\left(\phi_{s} \cos \theta+\psi_{s}\right)_{s}=2 K_{1}\left(\phi_{t} \cos \theta+\psi_{t}\right)_{t} \\
& K_{1} \phi_{t t} \sin \theta-2 K_{1} \theta_{t} \psi_{t}+\xi(s) \phi_{t} \sin ^{2} \theta= \\
& =A \phi_{s s} \sin \theta+2 A \phi_{s} \theta_{s} \cos \theta-C \theta_{s}\left(\phi_{s} \cos \theta+\psi_{s}\right)+F^{x} \sin \phi+F^{y} \cos \phi \\
& K_{1} \theta_{t t}+2 K_{1} \phi_{t} \psi_{t} \sin \theta+K_{1} \phi_{t}^{2} \sin \theta \cos \theta+\xi(s) \theta_{t} \\
& =A \theta_{s s}-A \phi_{s}^{2} \sin \theta \cos \theta+C \phi_{s} \sin \theta\left(\phi_{s} \cos \theta+\psi_{s}\right)-F^{z} \sin \theta-F^{x} \cos \theta \cos \phi+F^{y} \cos \theta \sin \phi,
\end{aligned}
$$

where $\xi(s) \phi_{t} \sin ^{2} \theta$ and $\xi(s) \theta_{t}$ are interpreted as being damping terms. One can then check that the total energy is decreasing with time i.e.

$$
\frac{d}{d t} E(\theta, \phi, \psi) \leq-\sigma \int_{0}^{1} \xi(s)\left(\phi_{t}^{2} \sin ^{2} \theta+\theta_{t}^{2}\right) d s<0
$$

where $\sigma>0$ is a positive coefficient that can be computed explicitly. In particular, this implies that the total energy can still be used as a suitable Liapounov function whilst studying dynamic stability [3]. We note that it is possible to obtain stronger stability results (asymptotic 
stability/exponential stability) with a strictly decreasing energy functional but we restrict ourselves to Liapounov stability throughout this paper $[2,24]$, for consistency with previous sections.

Proposition 6. The unbuckled equilibrium $\Theta_{0}$ defined in (5) is Liapounov stable in the set of twice-differentiable solutions, $\Theta^{*}(s, t)$, of the dynamical system (74) subject to the boundary conditions (3), for forces

$$
|F|<\min \left\{\pi^{2}(A-2 \pi M C), A \pi^{2}-2 \pi M C\right\} .
$$

Here, Liapounov stability is defined with respect to the norm defined in (15).

Comment: The condition (76) necessarily implies that $A>2 \pi M C$ in the presence of an external load $|F|>0$.

Proof: The proof is a minor modification of the proof of Proposition 4. The inequalities (46), (47) and (52) remain unchanged. Since the total energy is a decreasing function of time, the inequality (53) is modified to

$$
b_{1}\left\|\Theta_{1}(., t)\right\|^{2} \leq \Delta E(t)=\Delta V(t)+\Delta T(t) \leq \Delta E(0) \leq b_{2}\left\|\Theta_{1}(., 0)\right\|^{2}
$$

where $b_{1}, b_{2}$ are positive constants that only depend on $A, C, K_{1}, \rho,|F|$ and $M$. However, $\left\|\Theta_{1}(., 0)\right\|^{2}<\epsilon$ by assumption and hence,

$$
\left\|\Theta_{1}(., t)\right\|^{2} \leq \frac{b_{2}}{b_{1}} \epsilon
$$

for all $t \geq 0$, thus establishing the claimed Liapounov stability for forces

$$
|F|<\min \left\{\pi^{2}(A-2 \pi M C), A \pi^{2}-2 \pi M C\right\} .
$$

A similar result concerns the Liapounov stability of arbitrary minima of the potential energy $V$, in connection to the damped dynamical system (74).

Proposition 7. Let $\Theta^{*}=\left(\theta^{*}(s), \phi^{*}(s), \psi^{*}(s)\right)$ be an arbitrary extremal of the potential energy (6), subject to the clamped boundary conditions in (3), such that $\theta^{*} \in(0, \pi)$ for $s \in[0,1]$. Then $\Theta^{*}$ is Liapounov stable in the set of $C^{2}$-solutions of the dynamical system (74), with respect to the norm defined in (15), if

$$
\begin{aligned}
& \Gamma_{2}=\min _{s \in[0,1]}\left(A \sin ^{2} \theta^{*}-A\left|\phi_{s}^{*} \sin 2 \theta^{*}\right|-C\left|\cos \theta^{*}\right|-C K \sin \theta^{*}\right)>0 \\
& \Gamma_{3}=\min _{s \in[0,1]}\left(1-\left|\cos \theta^{*}\right|-\left|\phi_{s}^{*} \sin \theta^{*}\right|\right)>0 \\
& \Gamma_{1}=\min _{s \in[0,1]}\left(A\left(\phi_{s}^{*}\right)^{2} \cos 2 \theta^{*}-A\left|\phi_{s}^{*} \sin 2 \theta^{*}\right|-C\left|\phi_{s}^{*} \sin \theta^{*}\right|-C K \phi_{s}^{*} \cos \theta^{*}-C K \sin \theta^{*}\right)>-A \pi^{2}
\end{aligned}
$$

and for applied forces

$$
|F|<\min \left\{\pi^{2} \Gamma_{2}, A \pi^{2}+\Gamma_{1}\right\} .
$$


Proof: The proof of Proposition 7 parallels that of Proposition 6 above and the details are skipped for brevity.

Propositions 6 and 7 illustrate that stability results for conservative dynamical systems of the form (22) can be extended to damped systems of the form (74), provided we can construct a Liapounov function that is non-increasing with time [2]. In the examples above, the total energy plays the role of a suitable Liapounov function and we use the equivalence between the total energy and the norm, defined in (15), to establish Liapounov stability.

\section{Stability estimates for BVP II}

The clamped boundary conditions in BVP I are the simplest choice of boundary conditions. Nevertheless, the boundary conditions and the isoperimetric constraints in BVP II are of practical interest since they naturally arise in classical experiments with controlled enddisplacements. BVP II is technically harder than BVP I and the key difference arises from the inequalities, (11) and (12) which, in turn, affects the corresponding stability estimates as shown below. Our first result reproduces a well-known result for the stability of the trivial equilibrium state in two dimensions [17]. We obtain this result by a direct application of Wirtinger's inequality (see (12)) and present it here since it does not seem to have been previously reported in the literature.

\subsection{Static stability in two dimensions}

Proposition 8. Define the potential energy

$$
V[\phi, \lambda]=\int_{0}^{1} \frac{B}{2}\left(\frac{d \phi}{d s}\right)^{2}+\lambda \cos \phi d s
$$

where $\phi(s)$ denotes the angle between the tangent vector, $\mathbf{r}_{s}=\left(x_{s}, y_{s}\right)$, and the $x$-axis, $B>0$ is the bending stiffness of the rod and $\lambda$ is the external load. The boundary conditions are

$$
\phi(0)=\phi(1)=0
$$

accompanied by the fixed-end constraint

$$
\int_{0}^{1} \sin \phi(s) d s=0 .
$$

Then the trivial equilibrium, $\phi(s)=0$ for $s \in[0,1]$, is stable in the static sense for loads

$$
\lambda<4 B \pi^{2}
$$

and unstable for loads

$$
\lambda>4 B \pi^{2} .
$$


Proof: To demonstrate stability, it suffices to show that the second variation of $V[\phi, \lambda]$ about $\phi=0$ is strictly positive for loads $\lambda<4 B \pi^{2}$, for admissible perturbations [9, 17]. We consider perturbations of the form

$$
\phi_{\epsilon}(s)=\epsilon \eta(s)
$$

where

$$
\eta(0)=\eta(1)=0
$$

and

$$
\int_{0}^{1} \eta(s) d s=0 .
$$

The integral constraint above is a linearization of the nonlinear constraint, (82), about $\phi=0$. From [17], it suffices to consider the linearization of nonlinear constraints whilst computing the second variation; non-linear terms can be absorbed into the dominant linear term for small perturbations.

A direct computation shows that the second variation of $V[\phi, \lambda]$ about $\phi=0$ is given by

$$
\delta^{2} V[0, \lambda]:=\int_{0}^{1} B\left(\frac{d \eta}{d s}\right)^{2}-\lambda \eta^{2}(s) d s .
$$

Recalling (12), we have that

$$
\delta^{2} V[0, \lambda] \geq \int_{0}^{1}\left(4 B \pi^{2}-\lambda\right) \eta^{2}(s) d s
$$

and hence,

$$
\delta^{2} V[0, \lambda]>0
$$

for

$$
\lambda<4 B \pi^{2}
$$

as stated in (83).

To demonstrate instability, it suffices to construct a $\eta^{*} \in C^{1}([0,1] ; \mathbb{R})$, subject to the constraints above, for which $\delta^{2} V[0, \lambda]<0$. We take

$$
\eta^{*}(s)=\sin 2 \pi s .
$$

One can readily check that $\int_{0}^{1}\left(\frac{d \eta^{*}}{d s}\right)^{2} d s=4 \pi^{2} \int_{0}^{1}\left(\eta^{*}(s)\right)^{2} d s$. We substitute (87) into (85) to find that

$$
\delta^{2} V[0, \lambda]<0
$$

for $\lambda>4 B \pi^{2}$, as stated in (84).

Comment: The classical buckling load is, $\lambda=B \pi^{2}$, without fixed-end constraints as in (82). 


\subsection{Static stability in three dimensions}

Proposition 9. Consider the potential energy defined in (6)

$$
V[\theta, \phi, \psi]=\int_{0}^{1} \frac{A}{2}\left(\phi_{s}^{2} \sin ^{2} \theta+\theta_{s}^{2}\right)+\frac{C}{2}\left(\phi_{s} \cos \theta+\psi_{s}\right)^{2}+F \sin \theta \cos \phi d s
$$

subject to the clamped boundary conditions in (3) and the fixed-end constraints in (4). Then the unbuckled state $\Theta_{0}$, defined in (5), is stable in the static sense if $A>2 \pi M C$,

$$
|F|<\min \left\{4 A \pi^{2}-2 \pi M C, 4 A \pi^{2}-8 \pi^{3} M C\right\}
$$

and $M>0$ is the twist parameter defined in (3).

Proof: To demonstrate stability of the trivial equilibrium, we compute the second variation of the potential energy in (88). We consider arbitrary three-dimensional perturbations of the form

$$
\begin{aligned}
& \theta_{\epsilon}(s)=\frac{\pi}{2}+\epsilon \alpha(s) \\
& \phi_{\epsilon}(s)=\epsilon \beta(s) \\
& \psi_{\epsilon}(s)=2 \pi M s+\epsilon \gamma(s)
\end{aligned}
$$

where the $C^{1}$-functions, $\alpha, \beta, \gamma$, vanish at the end-points. Further, the functions $\alpha$ and $\beta$ are required to satisfy the linearization of the constraints (4) about $\Theta_{0}$, as shown below :

$$
\int_{0}^{1} \alpha(s) d s=0 ; \quad \int_{0}^{1} \beta(s) d s=0 .
$$

As in [18], we compute the second variation of the potential energy about $\Theta_{0}$ to obtain:

$$
\begin{aligned}
& \delta^{2} V\left(\Theta_{0}\right)=\left.\frac{d^{2}}{d \epsilon^{2}} V\left[\theta_{\epsilon}, \phi_{\epsilon}, \psi_{\epsilon}\right]\right|_{\epsilon=0}= \\
& =A \int_{0}^{1}\left(\frac{d \alpha}{d s}\right)^{2}+\left(\frac{d \beta}{d s}\right)^{2} d s+C \int_{0}^{1}\left(\left(\frac{d \gamma}{d s}\right)^{2}-4 \pi M \alpha \frac{d \beta}{d s}\right) d s-|F| \int_{0}^{1} \alpha^{2}+\beta^{2} d s \geq \\
& \geq \int_{0}^{1} A\left(\frac{d \alpha}{d s}\right)^{2}-(|F|+2 \pi M C) \alpha^{2} d s+\int_{0}^{1}(A-2 \pi M C)\left(\frac{d \beta}{d s}\right)^{2}-|F| \beta^{2} d s,
\end{aligned}
$$

where $\alpha$ and $\beta$ are constrained as in (91). Applying (12) to the integrals involving $\alpha, \beta$ above, we deduce that $\delta^{2} V\left(\Theta_{0}\right)>0$ for $|F|<\min \left\{4 A \pi^{2}-2 \pi M C, 4 A \pi^{2}-8 \pi^{3} M C\right\}$ as stated above. The positivity of the second variation for linearly admissible perturbations is sufficient to guarantee static stability $[3,17]$.

In [18], we demonstrate that $\Theta_{0}$ is stable in the static sense for forces

$$
|F|<\min \left\{\pi^{2}(A-2 \pi M C), A \pi^{2}-2 \pi M C\right\},
$$

for BVP I. As in Proposition 8, there are differences in the estimates for the buckling load in three dimensions, for BVP I and BVP II respectively. 


\subsection{Dynamic stability for BVP II}

Proposition 10. The trivial equilibrium $\Theta_{0}$, defined in (5), is Liapounov stable in the set of $C^{2}$-solutions, $\Theta^{*}(s, t)$, of the dynamical system (22) subject to the boundary conditions (3) and (4), if $A>2 \pi M C$ and for forces

$$
|F|<\min \left\{4 A \pi^{2}-2 \pi M C, 4 A \pi^{2}-8 \pi^{3} M C\right\} .
$$

Here, Liapounov stability is defined in terms of the norm (15).

Proof: The proof of Proposition 10 parallels that of Proposition 4 and is an immediate consequence of Proposition 9, the conservation of energy and the equivalence between the total energy and the norm defined in (15).

In [3], the authors address questions related to the equivalence between static and Liapounov stability for arbitrary planar equilibria, for boundary-value problems with integral constraints of the form (82). Here, we analyze the stability, both static and dynamic, of only the trivial equilibrium for BVP II, in a three-dimensional context. This is because we cannot apply simple integral inequalities of the form (12) to the linearized integral constraints around arbitrary equilibria and further extensions need to be considered.

\section{Conclusions}

We study the static and dynamic stability of arbitrary extremals of the general quadratic strain energy in (6), in a three-dimensional framework. In particular, we establish the equivalence between static stability and Liapounov stability under explicit conditions in Proposition 5. Our work heavily builds on the mathematical machinery in [3] and we generalize (to some extent) the two-dimensional results in [3] to three dimensions. As in [3], we use the direct method due to Liapounov, with the total energy as a suitable Liapounov function.

The main novelty of our work lies in the derivation of explicit stability criteria for arbitrary extrema, in Propositions 3 and 5. In the absence of body forces and couples, there are explicit formulae for the stationary equilibria in terms of elliptic functions; see [20]. The stability criteria in (25)-(26) can be explicitly computed for these elliptic functions and the bounds, (25)-(26), can be used to quantify the stability regimes of nearby equilibria, in the presence of a terminal load.

We introduce local damping forces in Section 5, analogous to local drag forces studied in the context of cellular biophysics, including bacterial flagellar motion and DNA transcription and replication $[13,23]$. The drag force in (70) is proportional to the local velocity of the tangent vector at each point of the Kirchhoff rod. It is useful to know that stability results for conservative systems hold for systems with such drag forces. The natural next step would be to consider non-local drag forces and study the dynamic evolution of local energy minimizers i.e. do the solutions remain confined to a small neighbourhood of the local energy minimizer for all times or can the dynamics drive the system out of equilibrium altogether.

In Section 6, we set up the mathematical machinery for stability analysis of constrained boundary-value problems in three dimensions. Our results are clearly only a first step and there are alternative, very successful approaches to stability analysis in the literature [10, 19, 
21]. However, our methods are direct and simple to use and there is scope for a successful generalization to a larger class of boundary conditions, a larger class of integral constraints (e.g. topological constraints as in [8]) and analysis of post-buckling behaviour in Kirchhoff rods.

Acknowledgements: AM is supported by an EPSRC Career Acceleration Fellowship, EP/J001686/1, and a Keble Research Fellowship, University of Oxford and is hosted by the Department of Mathematical Sciences, University of Bath. AM would like to thank the Oxford Center for Collaborative Applied Mathematics for its hospitality over the months of August-October 2012, during which this work was completed. This publication is based on work supported by Award No. KUK-C1-013-04, made by King Abdullah University of Science and Technology (KAUST), and based in part upon work supported by the National Science Foundation under grants DMS-0907773 (AG). AG is a Wolfson Royal Society Merit Holder and is supported by a Reintegration Grant under EC Framework VII. The authors thank John Maddocks for helpful discussions and for drawing their attention to the crucial role of polar singularities in the second variation analysis. The authors also thank Sebastien Neukrich for helpful discussions on isoperimetric constraints.

[1] S. S. Antman. Nonlinear problems of elasticity. Springer New York, 2005.

[2] J.M. Ball. Some open problems in elasticity. In Geometry, Mechanics, and Dynamics, 3-59, Springer, New York, 2002.

[3] R. E. Caflish and J. H. Maddocks. Nonlinear dynamical theory of the elastica. Proc. R. Soc. Edinb. A, 99, 1984.

[4] N. Chouaieb, A. Goriely, and J. H. Maddocks. Helices. Proc. Nat. Acad. Sci. USA, 103(25):9398-9403, 2006.

[5] B. D. Coleman, E. H. Dill, M. Lembo, Z. Lu, and I. Tobias. On the dynamics of rods in the theory of Kirchhoff and Clebsch. Arch. Rational Mech. Anal., 121:339-359, 1993.

[6] B. Dacorogna . Direct methods in the calculus of variations. Springer, 2008.

[7] L. Evans. Partial Differential Equations. American Mathematical Society, Providence, 1998.

[8] B. Fain, J. Rudnick and S. Ostlund . Conformations of linear DNA. Phys. Rev. E. 55 (6) : 7364-7367, 1997.

[9] M. R. Hestenes. Calculus of variations and optimal control theory. Wiley, 1966.

[10] K. A. Hoffman. Methods for determining stability in continuum elastic rod-models of DNA. Phil. Trans. R. Soc. Lond. A, 362:1301-1315, 2004.

[11] N. G. Hunt and J. E. Hearst. Elastic model of DNA supercoiling in the infinite length limit. J. Chem. Phys., 12:9329-9336, 1991. 
[12] H. Goldstein Classical Mechanics Addison-Wesley, Reading, Mass., Second Edition, 1980.

[13] R. E. Goldstein, T. R. Powers, and C. H. Wiggins. Viscous Nonlinear Dynamics of Twist and Writhe. Physical Review Letters, 80: 5232-5235, 1998.

[14] R. E. Goldstein and A. Goriely. Dynamic buckling of morphoelastic filaments. Phys. Rev. E, 74:010901, 2006.

[15] A. Goriely and M. Tabor. Nonlinear dynamics of filaments I: Dynamical instabilities. Phys. D, 105:20-44, 1997.

[16] A. Goriely and M. Tabor. Nonlinear dynamics of filaments II: Nonlinear analysis. Phys. D, 105:45-61, 1997.

[17] J. H. Maddocks. Stability of nonlinearly elastic rods. Arch. Ration. Mech. Anal., 85(4):311-354, 1984.

[18] A. Majumdar, C. Prior and A. Goriely. Stability estimates for a twisted rod under terminal loads: a 3D study. Journal of Elasticity, 1-19, March 2012.

[19] R.S. Manning, K.A. Rogers, and J.H. Maddocks. Isoperimetric conjugate points with application to the stability of dna minicircles. Proc. R. Soc. Lond. Ser. A Math. Phys. Eng. Sci., 454:3047, 1998.

[20] M. Nizette and A. Goriely. Towards a classification of Euler-Kirchhoff filaments. J. Math. Phys., 40:2830-2866, 1999.

[21] O. M. O'Reily and D. M. Peters. On Stability Analyses of Three Classical Buckling Problems for the Elastic Strut. J. Elast., 105:117-136, 2011.

[22] Y. Shi and J. E. Hearst. The Kirchhoff elastic rod, the nonlinear Schrödinger equation and DNA supercoiling. J. Chem. Phys., 101:5186-5200, 1994.

[23] C. W. Wolgemuth, T. R. Powers and R. E. Goldstein. Twirling and Whirling: Viscous Dynamics of Rotating Elastic Filaments. Physical Review Letters, 84: 1623-1626, 2000.

[24] H. Ziegler. Principles of structural stability. Waltham, Mass:Blaisdell, 1968. 



\section{RECENT REPORTS}

12/51 Meniscal tear film fluid dynamics near Marx's line Zubkov

Breward

Gaffney

12/52 Validity of the Cauchy-Born rule applied to discrete cellular-scale

Davit models of biological tissues

Osborne

Byrne

Gavaghan

Pitt-Francis

12/53 A thin rivulet or ridge subject to a uniform transverse shear stress

Sullivan at its free surface due to an external airflow

Paterson

Wilson

Duffy

12/54 The Stokes boundary layer for a thixotropic or antithixotropic fluid

McArdle

Pritchard

Wilson

12/55 Thermoviscous Coating and Rimming Flow

Leslie

Wilson

Duffy

12/56 On the anomalous dynamics of capillary rise in porous media

Shikhmurzaev

Sprittles

12/57 Compactly supported radial basis functions: how and why? Zhu

12/58 Multiscale reaction-diffusion algorithms: pde-assisted Brownian Franz dynamics

Flegg

Chapman

Erban

12/59 Numerical simulation of shear and the Poynting effects by the fi- Mihai nite element method: An application of the generalised empirical Goriely inequalities in non-linear elasticity

12/60 From Brownian dynamics to Markov chain: an ion channel example

Chen

Erban

Chapman

12/61 Three-dimensional coating and rimming flow: a ring of fluid on a

Leslie rotating horizontal cylinder

Wilson

Duffy

12/62 A two-pressure model for slightly compressible single phase flow in bi-structured porous media

Soulaine

Davit

Quintard

12/63 Mathematical modelling plant signalling networks

Muraro

Byrne

King

Bennett

12/64 A model for one-dimensional morphoelasticity and its application

Menon to fibroblast-populated collagen lattices

Hall

McCue

McElwain 
12/66 Morphoelastic Rods Part I: A Single Growing Elastic Rod

Moulton

Lessinnes

Goriely

12/67 Wrinkling in the deflation of elastic bubbles

Aumaitre

Knoche

Cicuta

Vella

12/68 Indentation of ellipsoidal and cylindrical elastic shells

Vella

Ajdari

Vaziri

Boudaoud

12/69 Memory of Recessions

Cross

McNamara

Pokrovskiif

12/70 An estimate of energy dissipation due to soil-moisture hysteresis

McNamara

12/71 The Mathematics Behind Sherlock Holmes: A Game of Shadows

Goriely

Moulton

12/72 Some observations on weighted GMRES

Güttel

Pestana

12/73 Bounds on the solution of a Cauchy-type problem involving a Furati weighted sequential fractional derivative

Copies of these, and any other OCCAM reports can be obtained from:

Oxford Centre for Collaborative Applied Mathematics

Mathematical Institute

24 - 29 St Giles'

Oxford

OX1 3LB

England

www.maths.ox.ac.uk/occam 